\title{
Education and training in integrated optics at the University of Twente
}

Rene de Ridder, Paul Lambeck, Hugo Hoekstra, Alfred Driessen

Rene M. de Ridder, Paul V. Lambeck, Hugo J.W.M. Hoekstra, Alfred Driessen, "Education and training in integrated optics at the University of Twente," Proc. SPIE 3190, Fifth International Topical Meeting on Education and Training in Optics, (8 December 1997); doi: 10.1117/12.294403 1997, Delft, Netherlands 


\title{
Education and training in Integrated Optics at the University of Twente
}

\author{
René M. de Ridder, Paul V. Lambeck, Hugo J.W.M. Hoekstra and Alfred Driessen \\ University of Twente, Departments of Electrical Engineering and Applied Physics \\ P.O. Box 217, 7500 AE Enschede, the Netherlands. \\ E-mail: R.M.deRidder@el.utwente.nl
}

\begin{abstract}
The curriculum in Electrical Engineering at the University of Twente has been recently adjusted in order to increase the proficiency in optics of the graduates, providing a general background and preparing especially for integrated optics and optical communication techniques. This involves mainly three undergraduate courses during the second through fourth year of the five-year curriculum. Two of these courses involve intensive use of computer aids. In the first one, Electrodynamics, Maple worksheets are extensively used for diminishing the tedium of the mathematics and for visualising (using animation) of travelling and standing wave patterns. In the last course, computer programs (a slab mode solver and an implementation of the Beam Propagation Method) are used as design tools. We describe the aims, contents, and the relationship between the courses and some organisational issues. It is concluded that the courses meet our requirements: undergraduate students become productive quite fast in the field of integrated optics when they work in an internship or in their MSc-project. The background thus provided to our graduates seems to be well received in the relevant industry.
\end{abstract}

Keywords: Education, Electrical Engineering, Optics, Planar Optical Waveguides, Integrated Optics, Computer Aided Design, Beam Propagation Method.

\section{INTRODUCTION}

Due to the importance of integrated optical functions for communications, sensors and signal processing, a formal undergraduate course in Integrated Optics was introduced a few years ago in both the departments of Electrical Engineering and Applied Physics of the University of Twente. This course is the basis for students wishing to extend their knowledge and skills in the field of integrated optics, by designing, fabricating and testing optical devices in the laboratories. Until that time, students wishing to do their MSc-thesis work in the field of integrated optics had a hard time in collecting the necessary introductory material for understanding the basic concepts and the more advanced material, e.g. for understanding and properly applying the analytical and numerical tools for designing planar optical devices. Especially for students of Electrical Engineering this was difficult, since, contrary to Applied Physics, there is no long standing tradition in optics in that field, and there are quite a few 'missing links'. Therefore, in this paper we will concentrate on the more extensive development of courses required for bringing students of Electrical Engineering to the required level of expertise.

We will first give a sketch of the current curriculum in Electrical Engineering (EE). Then, we will highlight (parts of) the courses in this curriculum that have special relevance to integrated optics. Next, we will give some examples of the teaching material used in these courses. Finally, we will mention a few results from thesis work done by students for their MSc. degree and for a postgraduate designers training, in order to give an impression of the level obtained.

\section{EE CURRICULUM}

The curriculum in Electrical Engineering at the University of Twente is oriented towards design and realisation of devices and systems that find their main application in information technology. The first three years are equal for all EE students. Here we find the usual foundation in mathematics (calculus, analysis, linear structures, probability, information theory, numerical computing), physics (electromagnetic field theory, mechanics, solid-state physics, optical basic functions), computer science and social science. Of course, a number of basic EE-courses are given (electronics, digital design, network theory, linear systems, physical systems theory, semiconductor devices, signal theory, measurement and control engineering, transmission media, modulation). Many of these courses are supported by lab work, either strongly prestructured or in a more free project form. There are special projects aimed at integrating the knowledge and skills obtained from different courses.

For the last two years, the students can choose between 3 prestructured variant streams: (1) Micro-electronics, transducers and microsystems, (2) Measurement and control engineering, and (3) Tele-informatics. Besides the required courses for the chosen stream, the students have some freedom in profiling themselves by taking at least 5 optional courses (from a list of 
approximately 70 courses). The fifth and final year is mainly devoted to an internship in industry and the thesis work (1000 hours) which must be defended for obtaining the Dutch "ingenieur" title (MSc.).

In the post-graduate phase, a one-year teachers training program, two-years designers programs and four-years $\mathrm{PhD}$ programs are available.

\section{COURSES RELATED TO INTEGRATED OPTICS}

Figure 1 shows the main relations in a cluster of courses in the department of Electrical Engineering related to (integrated) optics. Of course, many relations exist with other courses in the curriculum, which are omitted here for clarity.

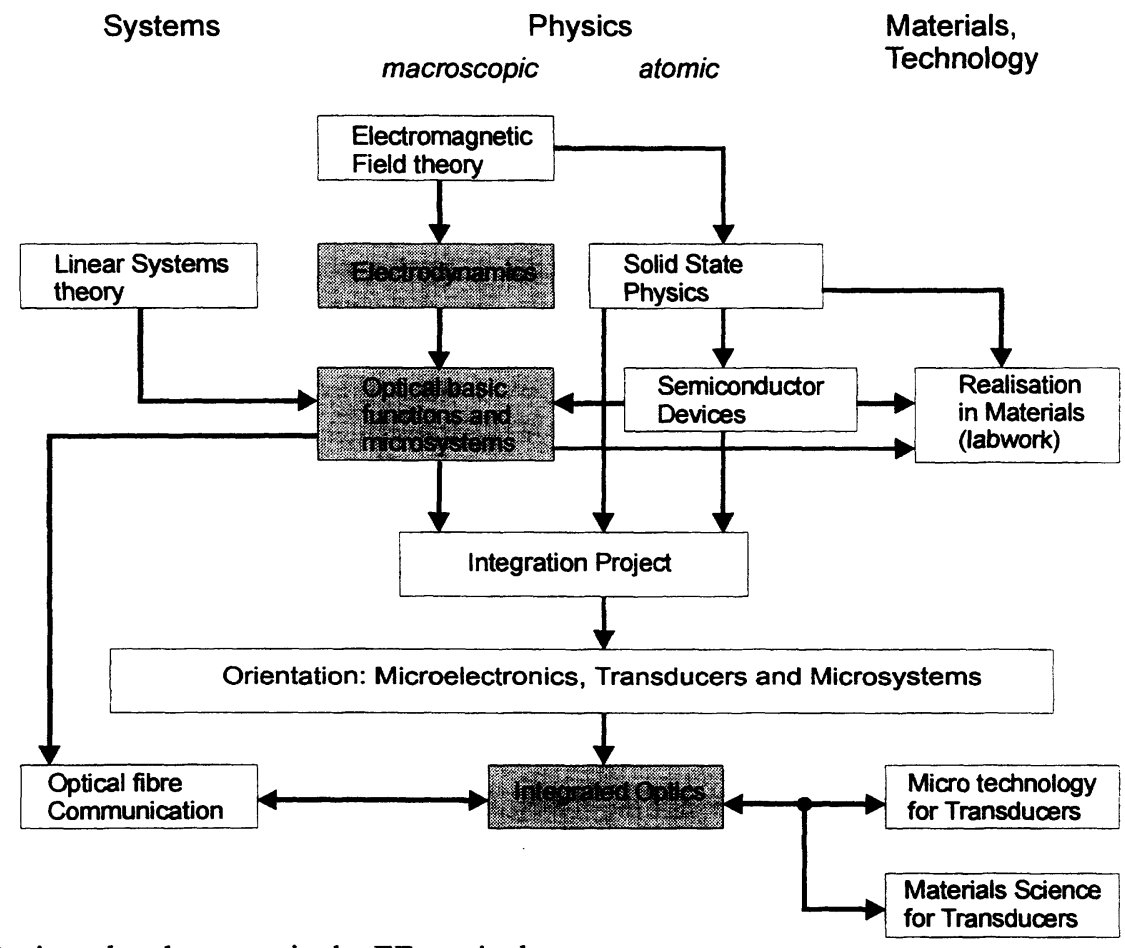

Fig. 1. Integrated Optics related courses in the EE curriculum.

In order to bridge the gap that originally existed between the basic EE curriculum and the final (optional) course in integrated optics, one course (Electrodynamics) had to be modified and another one (Optical basic functions and microsystems) was newly introduced. For Applied Physics, such a gap did not exist. Each of the courses mentioned below in 3.1 through 3.3 (the shaded blocks in fig. 1) has a formal study load of 100 hours for the students.

\subsection{Electrodynamics}

This second-year course builds on the first-year course in Electromagnetic Field theory where Maxwell's equations are introduced and their implications for (quasi-)static electric and magnetic fields in simple media are treated. In Electrodynamics, the treatment is extended to the time-dynamic regime, in particular the time-harmonic case. After discussing the radiating electrical dipole and plane waves in various simple media and their interaction with boundary planes between different media, considerable attention is given to waveguiding phenomena. Historically, emphasis was given to hollow conducting waveguides, especially the rectangular waveguide, and to radiating systems (antenna arrays). Dielectric waveguides were not mentioned at all.

The changes involve introducing the symmetrical dielectric slab waveguide and omitting details of rectangular waveguides and the entire subject of antenna arrays. Also, more attention is given to polarisation of plane and guided waves. The treatment of waveguiding is limited to two-dimensional systems (parallel plates, dielectric slab, coaxial cable) and both similarity and differences between waveguides in conducting and dielectric systems are emphasised. We retained the subject of transmission lines and the relation between reflections and impedance, since this is still of great importance for an electrical engineer's daily practice. 


\subsubsection{Course materials}

The same textbook by D.K. Cheng ${ }^{1}$ is used as in the preceding first-year course. Furthermore, a course manual is available containing some errata and addenda to the text book. The main issues here are a more thorough discussion of the quasi-static approximation, the relation between the ray model and the Maxwell treatment of dielectric waveguides, a simplified treatment of transmission lines and a generalisation of the amplitude reflection coefficient.

Computer-aided training facilities are integrated into the teaching. They take the form of $\mathrm{MapleV}^{2}$ work sheets in which examples are given, using equations in almost the same notation as they can be found in the textbook. Using the symbolic and numerical processing capacity of Maple, the tedium of the mathematical details is greatly reduced for the students, so that they can concentrate on visualising the field patterns and interpreting the results. Of particular value are the animations that can be created for visualising the propagation of waves in space and time, where also the behaviour of field vectors (polarisation) can be studied. In the example of figure 1, the situation is shown of a plane wave, obliquely incident on the interface between 2 media having different refractive indices. The left-hand picture shows both reflection and transmission, while the right-hand figure illustrates the phenomenon of total reflection. It is quite instructive to watch the evolution of these patterns in time.

When the students start, the worksheet reflects the situation shown in the left-hand side of figure 2 . They are asked to change the angle of incidence or the material properties and to predict what will happen. Also, they are asked to change the worksheet so that it visualises the other polarisation and to calculate the direction of power flow in the different regions of space.

Similar techniques are used for visualising wave propagation in parallel-plate and dielectric slab waveguides.
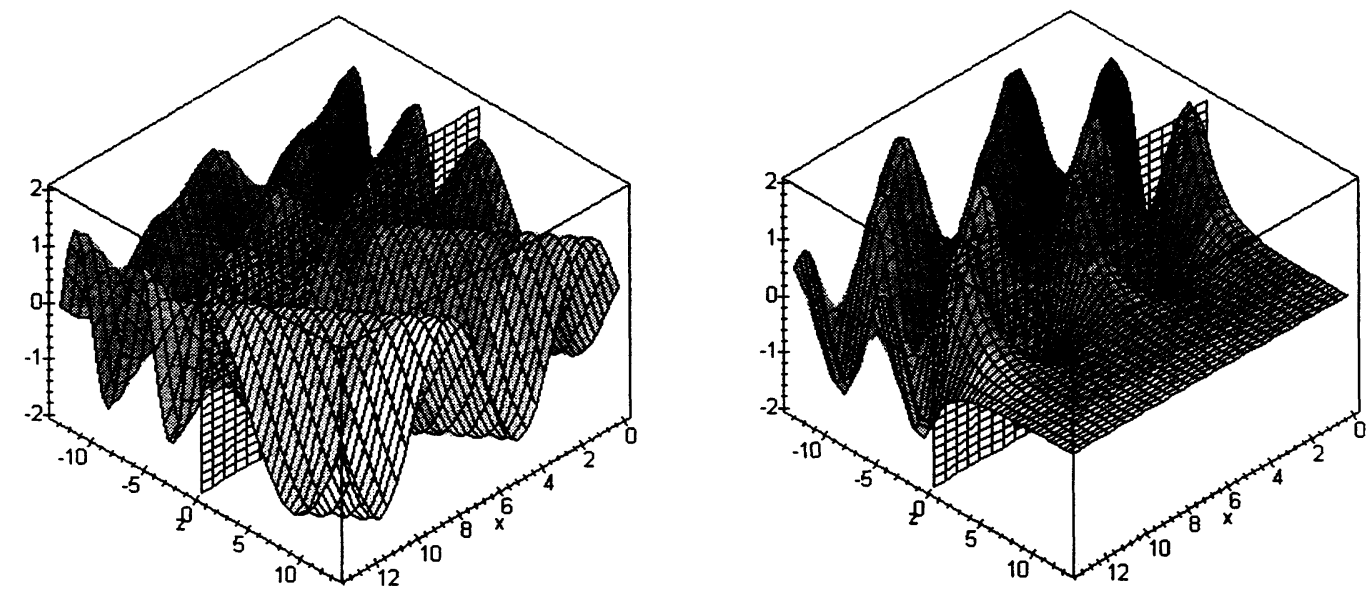

Fig. 2 Oblique incidence of plane wave on interface between two media. The 'tennis net' at $z=0$ represents the boundary plane between the media. The propagation vector of the s-polarised incident wave is in the $x-z$ plane, entering from 'behind' (from negative $x$ - and $z$-values). In the foreground we see the transmitted wave (left: plane wave coming towards the viewer; right evanescent surface wave). In the background (behind the 'net') we see an interference pattern formed by the incident and reflected waves. This pattern is a travelling wave in the direction parallel to the interface, and it is a standing wave in the direction perpendicular to it. The $y$-values in the figures show the momentaneous magnitude of the electric field.

\subsection{Optical basic functions and microsystems}

This is a new obligatory course in the third year, that was set up for three reasons: (1) deepening the understanding of linear transforms; (2) present some general knowledge on optics and optical components that is deemed indispensable for electrical engineers; and (3) demonstrating how knowledge on guided-wave phenomena can be applied for understanding principles of several components for integrated optics.

Towards the first aim mentioned above, scalar diffraction theory is used for calculating the transformation of optical wavefronts by a lens. It is shown that a lens can perform a two-dimensional Fourier transform almost instantly, and its applications in spatial filtering and image processing are briefly mentioned ${ }^{3,4}$.

The second aim is served with a treatment of Gaussian beams, again using diffraction theory to show how they propagate in free space and how they are transformed by lenses ${ }^{3}$. Furthermore, waveguiding in dielectric slabs ${ }^{5,6}$, channel waveguides ${ }^{6,7}$ and optical step-index fibres ${ }^{8}$ is reviewed. The Fabry-Perot interferometer ${ }^{9,10}$, and the principles of operation and some 
implementations of photo detectors, LED's, optical amplifiers and lasers are treated $9,11,12$. Before the introduction of this course, part of the material was presented in a (fourth year) optional course on optical fibre communication, but this was clearly too late, and moving it to the third year gave the opportunity to change the character of that course to a more in-depth treatment of advanced aspects of optical communication in the fourth year.

The subjects contributing to the third aim include multi-layer slab waveguides ${ }^{7}$, surface plasmon modes ${ }^{13}$, channel modes vs. system modes in devices such as directional couplers, multi-mode interferometers ${ }^{14}$, Y-junctions ${ }^{15}$, switches and waveguide input and output couplers ${ }^{16}$.

\subsubsection{Course materials}

Original material from various sources as given in the references to the preceding subsection, has been collected in a reader. Various introductions and explanations have been added, as well as treatments of the modes of a step-index fibre, local modes, system modes and adiabaticity and the rate equations in semiconductor lasers.

The material is visualised for the students by lecture-demonstrations, e.g. of spatial filtering of an image in the Fourier plane (figure 3) and an adjustable Fabry-Perot resonator.

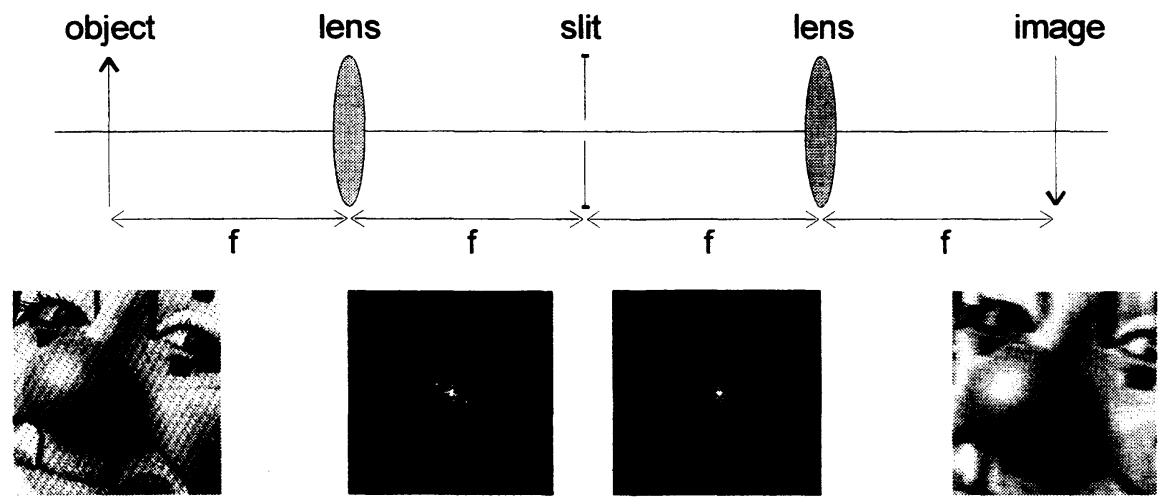

Fig. 3. Demonstration of filtering in the Fourier plane. The upper picture shows the experimental set-up. The first lens creates in its back focal plane a Fourier transform of the object in its front focal plane. A slit in the Fourier plane removes most high-spatial frequency components from the Fourier spectrum. The second lens performs the inverse transform to obtain the filtered image. On the lower row of the figure from left to right: original picture, its Fourier transform, the filtered Fourier transform and the filtered picture. (Note: for convenience, the pictures shown here have been created by a computer program. In the classroom experiment the slit can be rotated and its width is adjustable to show the 'real-time' filtering action.)

An interesting 'teaser' for the students is the demonstration of an optical fibre having fluorescent centres in its core. The fact that transversally incident radiation is converted to light that is guided by the fibre seems to violate the rule that such coupling is impossible.

\subsection{Integrated optics}

This course is obligatory for students in integrated optics and highly recommended for al other students following the variant stream on Micro-electronics, transducers and microsystems. Its main purpose is to provide the students with a solid background for participating in research projects and to make them acquainted with some major design tools used in this field.

First, the basic theory ${ }^{17}$ of dielectric waveguides based on the ray-picture and Maxwell's equations is briefly reviewed. The concept of phase-matching ${ }^{18}$ is illustrated first as following from the boundary conditions for plane waves transmitted through and reflected at the interface between different media. Then it is applied to the coupling between freely propagating and guided waves using prisms and gratings. Special attention is given to gratings operating in the Bragg regime. The phenomenon of mode conversion can be caused by abrupt changes in waveguide properties on the one hand and can be minimised when these properties are restricted to vary only slowly in the propagation direction (adiabatically) on the other hand. In this context, gratings are presented as efficient mode converters, providing both well-controlled waveguide disturbances and phase matching between selected modes. The method of attenuated total reflection (ATR) is discussed as an important application of prism coupling for the purpose of characterising optical properties of multi-layer structures. 
A number of analysis, design and simulation tools are discussed. The effective index method and the coupled mode theory, combined with a numerical slab solver are recommended for quick approximate solutions. The beam propagation method (BPM), which is a versatile tool for optimising and simulating the behaviour of (quasi) planar optical devices is explained ${ }^{19}$. The differences in applicability of various algorithms that may be implemented in a BPM simulator are pointed out.

A brief introduction to non-linear optics is given, covering a phenomenological description by introducing non-linear susceptibility tensors, some origins of non-linearity and their order of magnitude in media, and some of its applications. In particular, some attention is given to solitons.

An overview is given of important applications of integrated optics in the fields of optical communication and chemo-optical sensors ${ }^{20}$. Typical (sub)systems are shown, that are of interest in these fields and that are candidates for integration. Devices to be integrated in these systems are briefly mentioned, e.g. power splitters, modulators, switches, polarisation splitters, wavelength filters and (de)multiplexers, photo detectors, lasers, chemo-optical transducing elements, electronic circuits. A few of these examples ${ }^{21}$ are studied in more detail.

Examples of materials systems and the associated processing technologies are given ${ }^{22}$. More details are given on properties and required design tolerances of an optical thin-film technology ${ }^{23}$, available in our laboratory, that is compatible with standard silicon integrated circuit technology.

Finally, the students deepen their understanding by individually designing or optimising a relevant planar optical device, using the theory and computer aids (developed in our group) that were introduced earlier in the course. The students get marks for the course based on a defence of their designs.

\subsubsection{Course materials}

The students use a reader, in which parts of the standard material and some specific examples are taken from the literature, as indicated by the references to the previous subsection. Most of the rest of the needed written material was made specifically for this course.

Essential for this course is the use of computer programs for analysing, simulating and designing planar waveguide structures. First, there is a program called ATRGUIDE which, as its name may suggest, was primarily intended for analysing the results of prism-coupling and ATR measurements. It is, however, more than that: it contains a general multi-layer slab mode solver allowing for lossy materials (complex refractive index), and one of its most useful features is that it can quickly calculate how the effective indices of guided modes vary with a parameter of the waveguiding structure (thickness or refractive index of a particular layer). Besides the effective index or propagation constant, the program also determines the field distribution of the modes. An example of its use is shown in figure 4.

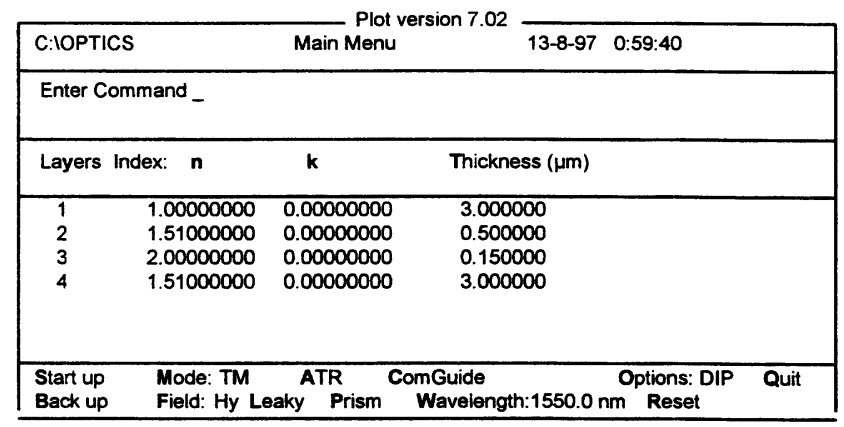

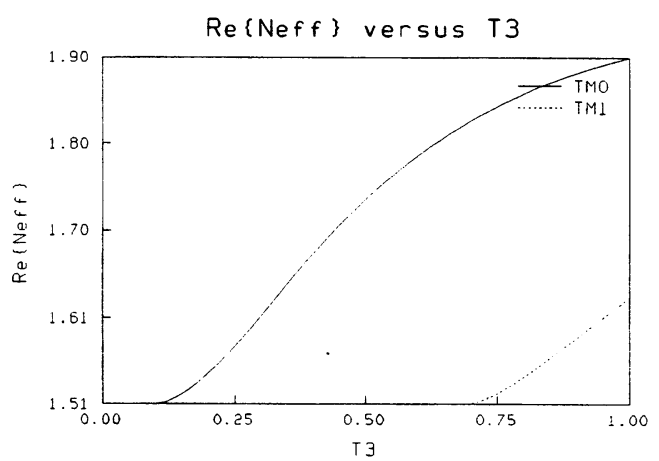

Fig. 4a. Example of using ATRGUIDE program: In the upper left the main menu is shown where the operating wavelength and the structure are defined (here a 4-layer structure of lossless materials). In the upper right we see the result of plotting the effective index of the guided modes versus the thickness of the highest-index layer 3 . There are 2 curves, showing that in the given range of values $\left(0 \leq t_{3} \leq 1 \mu \mathrm{m}\right)$ only $2 \mathrm{TM}$-modes can exist. For $t_{3} \leq 0.1 \mu \mathrm{m}$, no mode is guided and for $t_{3} \geq 0.7 \mu \mathrm{m}$ two guided modes exist. (Figure is continued on next page.) 

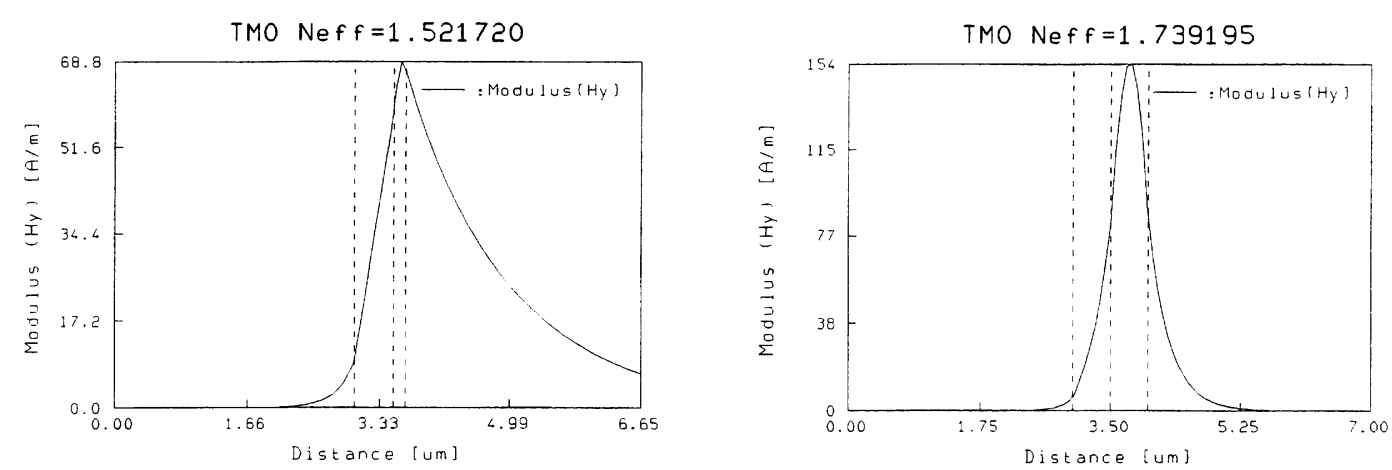

Fig. 4b. Students can easily calculate the distribution of the dominant field component of the fundamental TM-mode (the only existing mode) for $t_{3}=0.15 \mu \mathrm{m}$ (near cut-off) at the left hand side and the same mode, but now much better confined for $t_{3}=0.6 \mu \mathrm{m}$ at the right hand side. The dashed lines in the lower figures give the positions of the interfaces between the layers, from left to right as the definitions in the upper left picture run top to bottom.

The students can use this program to design a suitable layer stack and for example acceptable etching depth in the case that a ridge-type waveguide is to be used. Knowing the effective indices in the ridge region and outside, they can approximate the behaviour of a channel waveguide by using the effective index method.

Next, they can simulate the behaviour of a waveguide structure that changes in the direction of propagation (e.g. a Yjunction), using a well-documented BPM program called MENUFAST. Students get brief design tasks for which they get marks. Such a task could involve optimising a mode-splitting Y-junction with respect to cross-talk, see figure 5 .

Both programs ATRGUIDE and MENUFAST were developed in our group. They run with quite acceptable performance under MS-DOS on low-end personal computers.
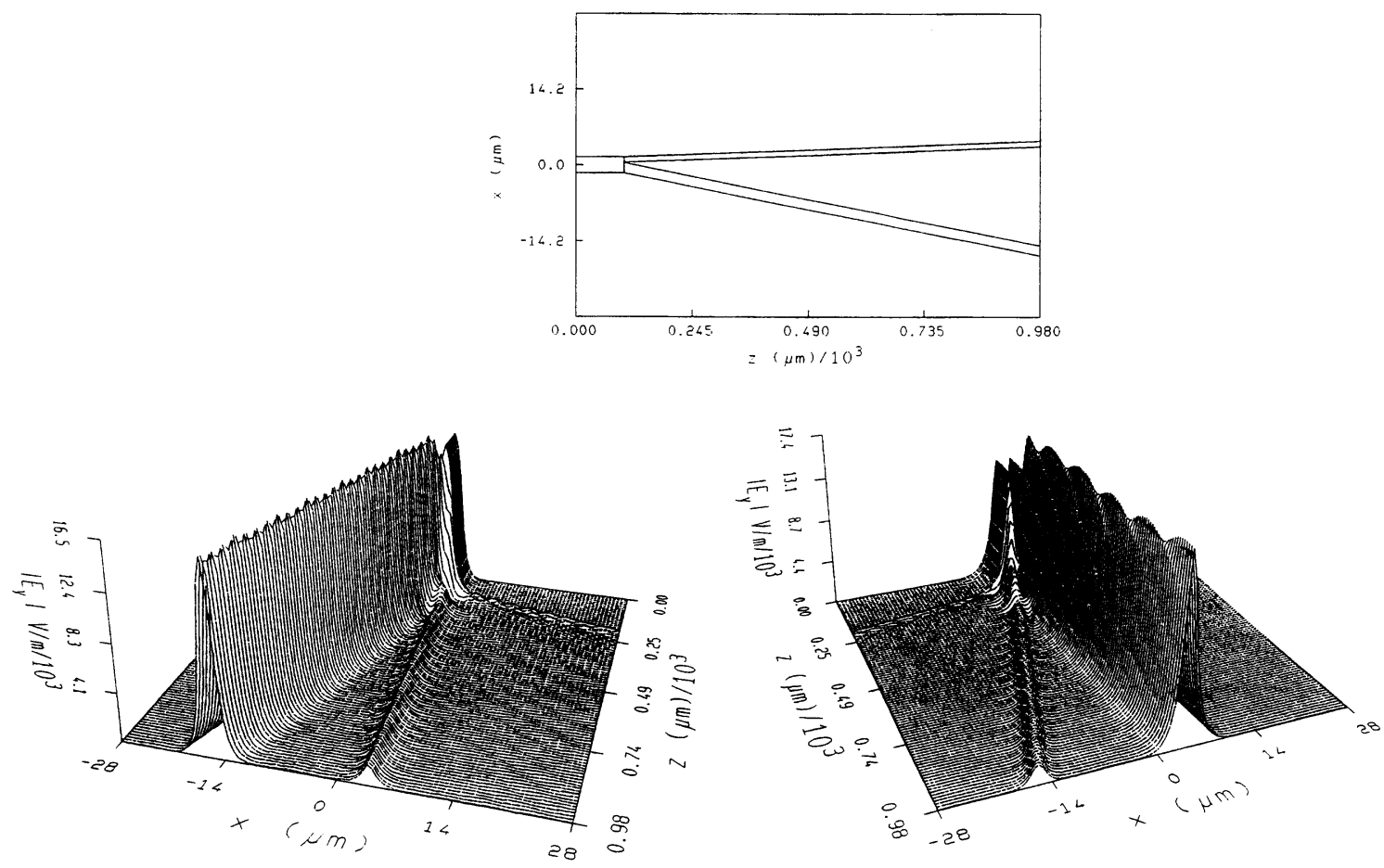

Fig. 5. Using the MENUFAST program to simulate the behaviour of an asymmetrical Y-junction (top). If the zero-order mode is launched at the input, most of its power will go into the widest output branch (left); most of the power launched in the first-order mode goes into the narrowest branch. 


\subsection{Other related courses}

We briefly mention three other courses that contribute to the competence of our students in integrated optics. The first one is a (third year) lab course called Realisation in Materials (40 hours study load), where students get hands-on experience in fabricating and characterising elementary devices in various fields. The students choose one out of four subjects: planar magnetic field sensor, micromechanical force sensor, planar optical humidity sensor or silicon photo detector. The students will experience the influence of processing parameters on device performance. Part of the work on the optical humidity sensor involves making a polymeric slab waveguide and characterising it using a prism coupling set-up.

An integration project (third year, 40 hours) aims at integrating the student's knowledge on optics, solid-state physics and semiconductor devices. A good example is the integration of a silicon photodetector with a planar optical waveguide.

At the end of the third year, a 200 hours course is intended to give all EE-students a taste of the variant stream Microelectronics, Transducers and Microsystems. The multidisciplinary development approach in this field is illustrated by studying several cases, including optical amplifiers and integrated optical sensors.

The course Microtechnology for Transducers (fourth year, 100 hours) gives an overview of deposition, etching and characterisation methods for thin-film devices.

Materials Science for Transducers (fourth year, 100 hours) presents selected topics (changing each year) that are of actual interest for either magnetic, micromechanical or optical transducers.

\section{OBTAINED LEVEL}

Most students that have taken the integrated-optics-oriented path through the curriculum perform very well, both internally in their MSc project and externally in industry (in internships and in normal jobs after graduation). MSc projects typically involve the design, realisation and characterisation of planar optical basic components or microsystems, such as a wavelength filter, a waveguide-system that has high sensitivity to environmental conditions (for sensor applications), an electro-optical or thermo-optical switch, a planar optical amplifier, or structures for efficiently coupling an optical fibre to a specific planar waveguide. We find that the students are provided with sufficient background to find quickly their way in the relevant technical literature and they are very soon up to speed with the design and analysis tools that are available. Besides the formal training described in the preceding section, the availability of a clean room laboratory with many advanced facilities for processing and characterising thin-film materials and the informal contacts with staff members and other students in the lab provide a very rich and stimulating environment where students can develop their talents and abilities.

Many of our graduates who already received this training (although in more experimental form) have found their way to companies where they are still active in the field of integrated optics or related fields. The reactions of employers to the obtained level are quite positive.

The same course in integrated optics is also offered to post graduate students, e.g. for our designers training. The bulk of the time for such a training goes into a design project. The subjects are similar to those mentioned as MSc projects, although the devices may be somewhat more complicated. The major difference is that the design process is driven by external specifications with emphasis on fabrication tolerances and taking into account the economic aspects of manufacturing such devices. A very nice example is the $8 \times 8$ polymeric switching matrix that was designed and realised by $\mathrm{A}$. Borreman ${ }^{24}$ during his designers project that was carried out externally at Akzo Nobel.

\section{ACKNOWLEDGEMENTS}

We gratefully acknowledge the contributions of many students, who helped us with their critical remarks to improve our lectures and course materials. Furthermore, many staff members and $\mathrm{PhD}$ students provided us with material that in some form found its way into the lecture notes, readers, computer aids or classroom experiments. In particular, we like to thank Hans Albers, Jos van Gent, Anton Hollink, Marcel Klein Koerkamp, Ton Koster, Gijs Krijnen, Jan van Schoot, Marcel Rijnsburger, Henk Vos and Henk van Wolferen. 


\section{REFERENCES}

1. D. K. Cheng, Field and Wave Electromagnetics, Addison-Wesley, Reading, $2^{\text {nd }}$ edition, 1989.

2. Maple V r 4, Waterloo Maple Inc., Waterloo, Ontario, Canada.

3. R. Syms, J. Cozens, Optical guided waves and devices, pp. 72-90, McGraw Hill, London, 1992.

4. G.O. Reynolds, J.B. Velis, G.B. Parrent jr., B.J. Thompson, The new physical optics notebook: Tutorials in Fourier optics, pp. 9-13 + 137-138, SPIE Optical Engineering Press, Bellington, 1989.

5. H. Kogelnik, "Theory of dielectric waveguides", Integrated Optics, T. Tamir (ed.), pp. 15-25 + 34-41, Springer, Berlin, 1979.

6. R.G. Hunsperger, Integrated Optics: Theory and Technology, pp. 31-43, Springer, Berlin, 1984.

7. G. Krijnen, All-optical switching in nonlinear integrated optic devices , pp. 19-48, PhD thesis, University of Twente, Enschede, 1992.

8. K. Iizuka, Engineering Optics, pp. 342-350, Springer, Berlin, 1985.

9. R. Syms, J. Cozens, Optical guided waves and devices, pp. 92-97, McGraw Hill, London, 1992.

10. B.J. Offrein, The Fabry-Perot resonator as an all-optical switching device, pp. 21-26, PhD thesis, University of Twente, Enschede, 1994.

11. J.M. Senior, Optical fiber communications: principles and practice, pp. 282-296, Prentice Hall, New York, 1992.

12. Kwok K. Ng, Complete guide to semiconductor devices, pp. 372-408, McGraw Hill, New York, 1995.

13. J. van Gent, Surface plasmon based chemo-optical sensors, pp. 15-19, PhD thesis, University of Twente, Enschede, 1990.

14. M.K. Smit, Integrated Optics in silicon based aluminium oxide, pp. 155-164, $\mathrm{PhD}$ Thesis, TU Delft, Delft, 1991.

15. G. Krijnen, All-optical switching in nonlinear integrated optic devices, pp. 133-136, PhD thesis, University of Twente, Enschede, 1992.

16. T. Tamir, "Waveguide input and output couplers", Integrated Optics, T. Tamir (ed.), pp. 88-90 + 94-99, Springer, Berlin, 1979.

17. H. Kogelnik, "Theory of optical waveguides", Guided-Wave Optoelectronics, T. Tamir (ed.), pp. 9-27, Springer, Berlin, $2^{\text {nd }}$ edition, 1990.

18. R. Syms, J. Cozens, Optical guided waves and devices, pp. 145-177, McGraw Hill, London, 1992.

19. H.J.W.M. Hoekstra, "On beam propagation methods for modelling in integrated optics", Optical and Quantum Electronics, 29, pp. 157-171, 1997.

20. P.V. Lambeck, "Integrated opto-chemical sensors", Sensors and Actuators B, 8, pp. 103-116, 1992.

21. R.M. de Ridder, A.F.M. Sander, A. Driessen, J.H.J. Fluitman, "An integrated optic adiabatic TE/TM mode splitter on silicon", J. Lightwave Technol., 11(11), pp. 1806-1810, 1993.

22. H. Albers, "Thin film technology for optical devices", Sensors \& Actuators: Optical Transducers, pp. 163-174, A. Driessen (ed.), Kluwer, Deventer, 1990.

23. W.S. Bijlsma, H. Albers, P.V. Lambeck, Th.J.A. Popma, "PECVD $\mathrm{SiO}_{\mathrm{x}} \mathrm{N}_{\mathrm{y}}$ films as planar optical waveguides" , Sensors \& Actuators: Optical Transducers, pp. 233-236, A. Driessen (ed.), Kluwer, Deventer, 1990.

24. A. Borreman, T. Hoekstra, M. Diemeer, H. Hoekstra, P. Lambeck, "Polymeric 8x8 digital optical switch matrix", Conf. Proc. ECOC'96, post-deadline paper, 1996. 